\title{
Mitteilungen des Deutschen Zentrums für Lungenforschung
}

Verantwortlich: Sabine Baumgarten (Redaktionsleitung), Dr. Jörn Bullwinkel, Dr. Birgit Teucher, Dr. Annegret Zurawski

Beim diesjährigen DGP-Kongress, bei dem das DZL erneut mit zahlreichen Preisträgern, Beiträgen sowie Infostand vertreten war, würdigte auch Bundesgesundheitsminister Hermann Gröhe das DZL als „erfolgreichen Zusammenschluss translationaler Forschung zur Bekämpfung von Lungenerkrankungen“. Ende Oktober erfolgte nun die Evaluation des DZL. Die Ergebnisse und die sich daraus ergebenden weiteren Entwicklungsmöglichkeiten für den Verbund werden mit großer Spannung erwartet. Einen Überblick über die Highlights des vergangenen Jahres und Forschungsergebnisse des DZL seit seiner Gründung können Sie im Jahresbericht 2014 nachlesen (online verfügbar via: http://www.dzl.de/index.php/en/pressand-events/downloads). Eindrücke des ERS-Kongresses 2015 und des 4. Internationalen DZL-Symposiums finden Sie ebenso in der aktuellen Ausgabe der Mitteilungsseiten wie Berichte über ausgewählte Forschungserfolge und -entwicklungen, Patientenforen und die Portraits zweier engagierter Nachwuchsforscherinnen.

\section{DZL beim ERS International Congress in Amsterdam $\nabla$}

Das DZL war auch in diesem Jahr beim International Congress der European Respiratory Society (ERS) mit Vortragenden, Preisträgern und einem eigenen Infostand vom 26.-30. September 2015 in Amsterdam vertreten. Beim weltweit größten Lungenkongress trafen sich mehr als 22000 Teilnehmer aus über 127 Ländern. $\mathrm{Zu}$ den ERS-Preisträgern zählten erneut auch DZL-Wissenschaftler. So ist Prof. Dr. Antje Prasse (DZL-Standort BREATH) für ihre Leistungen und ihre erfolgreiche Forschungsarbeit im Bereich der idiopathischen Lungenfibrose mit dem „ERS Research Award on Idiopathic Pulmonary Fibrosis 2015“ ausgezeichnet worden. Dr. Rajkumar Savai (DZL-Standort UGMLC) erhielt den „Sir John Vane Grant for Best Recent Publication in Pulmonary Vascular Research 2015" für seine Nature-Medi-

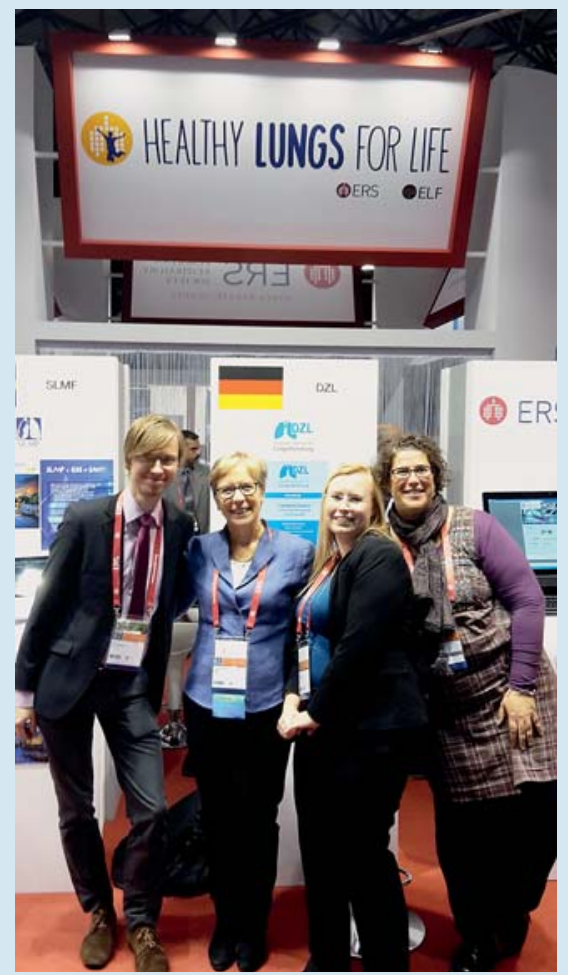

Abb.1 DZL-Vertreter mit ERS-Präsidentin E. Bel (v.l. n. r.: J. Bullwinkel, E. Bel, S. Baumgarten, A. Brand (Foto: DZL).

cine-Publikation „Pro-proliferative and inflammatory signalling converge on FoxO1 transcription factor in pulmonary hypertension“ (Nat Med. 2014 Nov; 20 (11): 1289-1300). Der „Presidential Award 2015“ ging an Prof. Jeffrey M. Drazen, eines der Mitglieder des Wissenschaftlichen Beirats des DZL und Editor-in-chief des „New England Journal of Medicine“ (NEJM). Er wurde damit für seinen herausragenden Beitrag zur Stärkung der weltweiten Lungenmedizin gewürdigt. Darüber hinaus verlieh die ERS zum zweiten Mal den Titel „Fellow of ERS (FERS)“ an exzellente Lungenforscher und -kliniker aus den Reihen des DZL erhielten diesen im Jahr 2015 Prof. Dr. Erika von Mutius (DZL-Standort CPC-M) und Prof. Dr. Roland Diel (DZL-Standort ARCN).

Im neu gestalteten Kongressbereich „World Village“ informierte das DZL gemeinsam mit anderen professionellen Fachverbänden aus aller Welt mit eige-

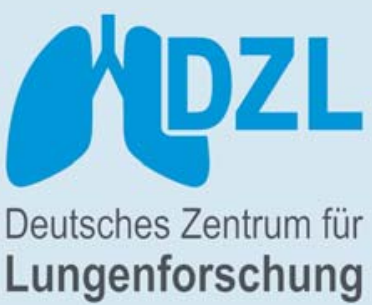

nem Stand über seine Aktivitäten und empfing die ERS-Präsidentin Prof. Dr. Elisabeth Bel beim alljährlichen „Presidents' Visit“. Mit der weltweiten Aufklärungskampagne „Healthy Lungs for Life“, die dieses Mal unter dem Motto „Take The Active Option“ lief, riefen ERS und ELF (European Lung Foundation) vor Ort schon auf, sich ein Pedometer ,zu schnappen' und mindestens 10000 Schritte am Tag zu gehen. Wie wichtig Bewegung ist, um die Lungengesundheit $\mathrm{zu}$ erhalten bzw. zu verbessern, zeigte die ELF-ERSKampagne zudem in verschiedenen Beiträgen im Kongressprogramm und mit Aktionen für die ganze Bevölkerung. Neu im Veranstaltungsprogramm waren die gutbesuchten Live-Endoskopie-Sessions zu verschiedenen Lungenkrankheiten. Das DZL plant auch im Jahr 2016 wieder beim ERS-Kongress in London dabei zu sein.

\section{Lungenforscher aus aller Welt} trafen sich beim 4. Internationalen DZL-Symposium in Heidelberg $\nabla$

Rund 200 Lungenforscher kamen beim 4. Internationalen DZL-Symposium/TLRC Lung Conference vom 25.-27. Juni in Heidelberg zusammen. Unter dem Motto „Frontiers in Chronic and Malignant Airways Disease“ widmete sich die Konferenz neuesten Forschungserkenntnissen zu chronischen sowie bösartigen Atemwegserkrankungen wie Mukoviszidose, COPD und Lungenkrebs. Im Fokus des Symposiums stand die Strategie, Überschneidungen in Krankheitsmechanismen zu erkennen, um innovative, krankheitsübergreifende Behandlungsansätze zu entwickeln. In den vier Plenar-Sessions „Cystic Fibrosis meets COPD“, „Mucus and the Microbiome“, „Molecular and Cell Biology of Lung Diseases“ und „Lung Cancer“ stellten international führende Experten neueste Erkenntnisse aus den Bereichen Grundlagenforschung, translationale Forschung und klinische Anwendung vor. So sprachen beispielsweise Professor Stuart Elborn von der Queens University Belfast 


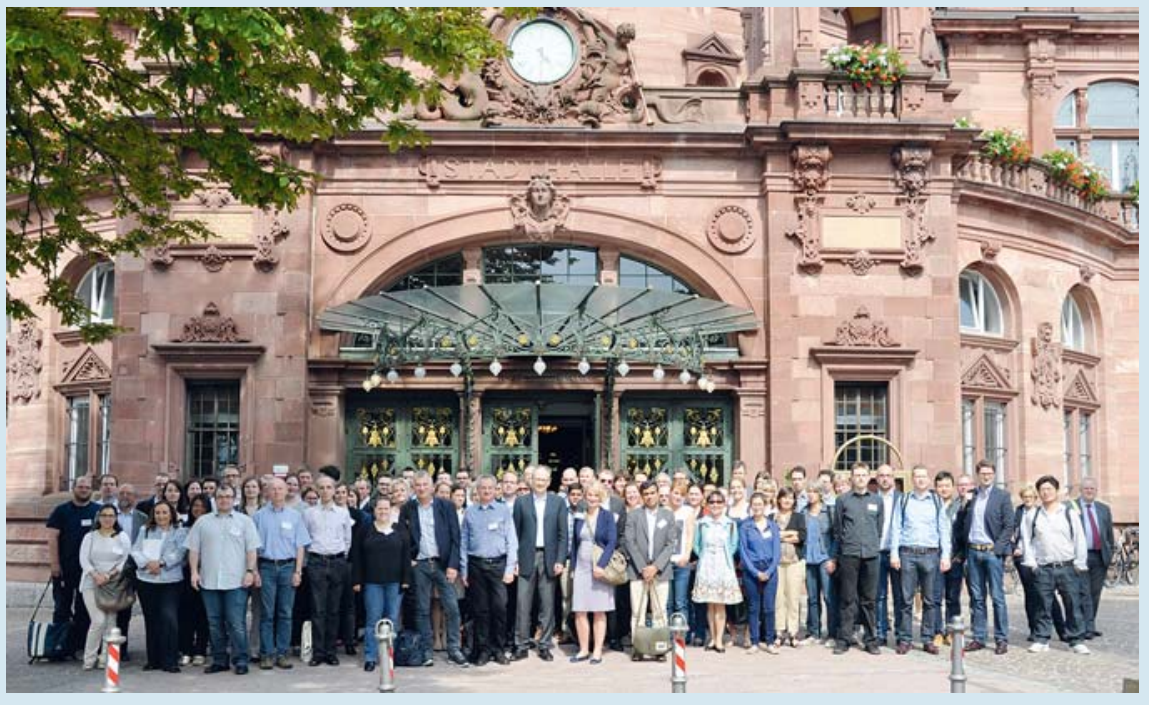

Abb. 2 4. Internationales DZL-Symposium in Heidelberg (Foto: DZL).

(UK), Mitglied im Internationalen Wissenschaftlichen Beirat des DZL, und Professor David J. Thornton von der University of Manchester (UK) über die Rolle des Mukus und des Mikrobioms bei chronischen Lungenerkrankungen. Zwei „Hot Topic“Vorträge beleuchteten darüber hinaus die Themen „Bildgebende Verfahren“ und „Systemmedizin“ in der Lungenforschung. Neben renommierten Wissenschaftlern erhielten auch junge Forscher erneut $\mathrm{Ge}$ legenheit, ausgewählte Beiträge in Kurzvorträgen dem Plenum vorzustellen und ihre Projekte in drei Postersessions zu präsentieren; drei DZL-Posterpreise wurden vergeben. Ausgerichtet wurde das Symposium in diesem Jahr vom DZLStandort Translational Lung Research Center Heidelberg (TLRC). Die Abstracts des Symposiums finden Sie in der Juliausgabe der Pneumologie.

\section{Bewusstsein für Bronchiektasen stärken - DZL unterstützt Register PROGNOSIS und EMBARC \\ $\nabla$}

Seit Juni dieses Jahres rekrutiert das deutsche Bronchiektasen-Register PROGNOSIS (The Prospective German Non-CF-Bronchiectasis Registry), das unter anderem von Klinikern des DZL-Standorts BREATH (Hannover) initiiert wurde, die ersten Patienten. In das Register werden erwachsene Patienten mit Bronchiektasen eingeschlossen, die nicht durch eine Zystische Fibrose (CF) verursacht wurden. PROGNOSIS will wichtige, bisher noch nicht verfügbare, epidemiologische Fragestellungen, wie u.a. die Alters- und Ge- schlechterverteilung, die Krankheitsentstehung, die Mikrobiologie und Behandlungsformen beantworten. „Auf Basis der Ergebnisse möchten wir unter anderem deutschsprachige Leitlinien für die Diagnostik sowie Prognosemarker für NonCF-Bronchiektasen entwickeln, um die Versorgung unserer Patienten nachhaltig zu verbessern“, so DZL-Wissenschaftler Dr. Felix Ringshausen, Vorstandsmitglied von PROGNOSIS.

Noch vor Kurzem wurden Bronchiektasen im European Lung White Book als eine der am meisten vernachlässigten Krankheiten weltweit bezeichnet. Non-CFBronchiektasen sind jedoch häufiger als bislang angenommen - die Prävalenz lag im Jahr 2013 in Deutschland bei 6,7 auf
10000 Personen. Somit zählen Non-CFBronchiektasen nach EU-Definition nicht zu den seltenen Erkrankungen.

Derzeit gibt es allerdings noch keine zugelassene medikamentöse Therapie; Ressourcen zur weiteren Erforschung der Erkrankung sind knapp. Mit der Gründung von EMBARC (The European Multicentre Bronchiectasis Audit and Research Collaboration) im Jahr 2012 und dem Start der Patientenrekrutierung für das europäische Register hat die Erkrankung höhere Aufmerksamkeit erlangt. PROGNOSIS wird seine Patientendaten in $\mathrm{Zu}-$ kunft nun ebenfalls in EMBARC einbringen, um diese auch im europäischen Kontext erfassen und analysieren zu können.

Unterstützung findet PROGNOSIS sowohl beim DZL-Partner CAPNETZ STIFTUNG, der mit dem Aufbau von Registern große Erfahrung hat, als auch beim DZL-Standort BREATH, der im kommenden Jahr die erste „EMBARC World Bronchiectasis Conference" ausrichten wird. Die Konferenz wird vom 7.-9. Juli 2016 im Schloss Herrenhausen in Hannover stattfinden. Eine Anmeldung zur Teilnahme sowie das Einreichen von Abstracts für Vorträge und Posterbeiträge ist bereits möglich unter: www.world-bronchiectasis-conference.com

Weitere Informationen:

www.bronchiektasen-register.de www.bronchiectasis.eu

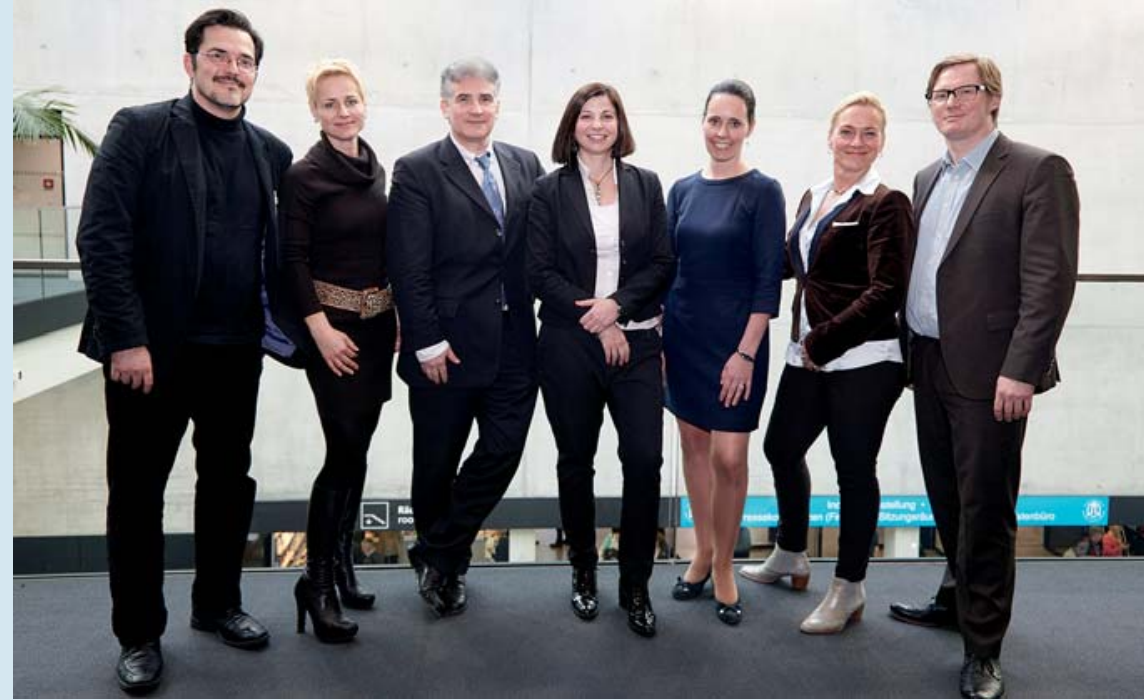

Abb. 3 Das PROGNOSIS-Team v.I.n. r.: A. de Roux, A. Zurawski, R. Diel, E. Polverino, J. Rademacher, G. Barten, F. Ringshausen (Foto: PROGNOSIS/DZL). 


\section{Besucherrekord beim COPD- Patientenforum in Großhansdorf $\nabla$}

Gemeinsam mit dem Lungeninformationsdienst veranstaltete die LungenClinic Grosshansdorf im April erstmals ein „Patientenforum Lunge“ am nördlichsten DZL-Standort, dem Airway Research Center North. Unter dem Motto „COPD Leben mit einer chronischen Erkrankung“ informierten Ärzte über Ursachen, Behandlungsmethoden sowie neue Forschungsstrategien. Mit Tipps und Übungen zu Atemtechniken kam auch die Praxis nicht zu kurz. Der Vortragssaal der LungenClinic war mit 150 Personen prall gefüllt, was einen neuen Besucherrekord in der erfolgreichen Reihe „Patientenforum Lunge“ darstellte. Da der Informationsbedarf in der Bevölkerung offenkundig groß ist, sollen zukünftig weitere Patientenforen in Großhansdorf und an anderen DZL-Standorten stattfinden. So luden der Lungeninformationsdienst und das Klinikum der Universität München (DZLStandort CPC-M) am 21. Oktober 2015 bereits zum 12. Patientenforum ein. Thema der Veranstaltung war „Asthma im Kindes- und Erwachsenenalter“.

\section{Erste BREATH Summer School begeistert Teilnehmer und Vortragende \\ $\nabla$}

Unter dem Thema „The failing lung - environmental damage and clinical repair" fand vom 10.-13. Juni 2015 mit finanzieller Unterstützung der VolkswagenStiftung die erste Summer School des DZLStandorts BREATH (Hannover) mitten im Naturschutzgebiet Harz statt. Zur Summer School hatte BREATH Doktoranden, Postdocs und Ärzte aller DZL-Standorte eingeladen. Den 21 Teilnehmern wurde ein abwechslungsreiches Programm rund um wissenschaftliche Themen, Softskill-Kurse und Teamarbeit, Vorträge und Gespräche über Karrieren innerhalb und außerhalb der Universität sowie ein Outdoor-Teambuilding-Event geboten. International renommierte Gastredner trugen über ihre Forschungsarbeiten zu Schädigungen der Lunge vor. Einblicke in Erkrankungen wie Lungenfibrose, Bronchiektasen und Lungenerkrankungen im Endstadium gaben Ärzte und Wissenschaftler der Medizinischen Hochschule Hannover. Weitere Themen waren Reparaturmöglichkeiten mittels Stammzellen, der Ersatz der Lunge durch Extracorporeale Membranoxygenierung (ECMO) oder die Biohybridlunge.

\section{$a z$}

\section{Reihe „DZL-Nachwuchswissen- schaftler im Profil" $\nabla$}

Neben Summer und Autumn Schools an den verschiedenen DZL-Standorten stand das Thema "Nachwuchsförderung" auch im Fokus der diesjährigen Panel-Diskussion der Deutschen Zentren der Gesundheitsforschung (DZG), die am 13. Oktober im Rahmen des World Health Summit unter dem Motto „Education and Training of Clinical and Translational Scientists Different Models in Different Countries" in Berlin stattfand.

In dieser Ausgabe möchten wir Ihnen die DZL-Nachwuchsgruppenleiterin Prof. Dr. Susanne Herold von der Justus-LiebigUniversität Gießen (DZL-Standort UGMLC) und die Nachwuchswissenschaftlerin Prof. Dr. Rocio Sotillo vom Deutschen Krebsforschungszentrum in Heidelberg (DZL-Standort TLRC) vorstellen.

\section{Susanne Herold - „Das DZL bildet} eine ideale Plattform für die translationale ,bench-to-bedside‘Pipeline."

Im Sommer 2015 trat Susanne Herold die DZL-Professur und Nachwuchsgruppenleitung im Bereich „Acute Lung Injury“ an. Sie studierte Medizin an der JustusLiebig-Universität Gießen (JLU) und begann ihre Ausbildung als Assistenzärztin mit Schwerpunkt Infektiologie, Pneumologie und Intensivmedizin. Sie promovierte 2002 zum Dr. med., absolvierte im Rahmen des MD/PhD-Tracks das Graduiertenkolleg „Molecular Biology and Medicine of the Lung“" (MBML) und promovierte 2008 zum Ph.D. Seit 2010 ist sie Fachärztin für Innere Medizin, Infektiologin und Oberärztin im Schwerpunkt Infektiologie. Im Jahr 2013 habilitierte sie sich an der JLU im Fach Innere Medizin. Neben ihrer Mitgliedschaft im Deutschen Zentrum für Lungenforschung gehört Herold dem Leitungsgremium des University of Giessen and Marburg Lung Center (UGMLC) und dem Vorstand der Deutschen Gesellschaft für Infektiologie (DGI) an. Sie ist außerdem Mitglied des Exzellenzclusters Cardio Pulmonary System (ECCPS), des Deutschen Zentrums für Infektionsforschung (DZIF) und zweier Sonderforschungsbereiche auf dem Gebiet der angeborenen Immunabwehr der Lunge sowie der Biologie von RNA-Viren.

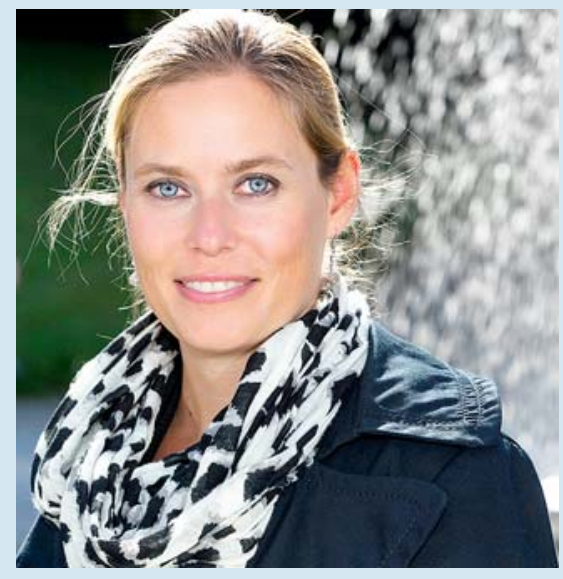

Abb. 4 DZL-Nachwuchsgruppenleiterin Prof. Dr. Susanne Herold (Foto: Laackman Fotostudios Marburg).

In ihrer Forschung beschäftigt sich Herold mit der Bedeutung und funktionellen Diversität des Monozyten-MakrophagenSystems bei Lungenentzündung mit unterschiedlichen Erregern wie zum Beispiel Influenza-Viren. Ihre Arbeiten adressieren sowohl die Rolle inflammatorischer Makrophagenpopulationen in der Progression des akuten Lungenversagens als auch die Funktion gewebe-regenerativer Makrophagen in der Entzündungsauflösung und der Stammzell-vermittelten Organregeneration nach akutem Lungenschaden. „Die Definition der molekularen Mechanismen und involvierten Mediatoren wird zukünftig Ausgangspunkt für translationale Therapieansätze und klinische Studien sein, um wirksame adjunktive Therapien für Patienten mit Pathogen-induziertem Lungenversagen zu etablieren. Insbesondere vor dem Hintergrund der zunehmenden Antibiotikaresistenzen bzw. fehlender Therapieoptionen bei viralen Infektionserkrankungen der Lunge sind solche Therapien dringend notwendig. Das Deutsche Zentrum für Lungenforschung bildet für die translationale ,bench-to-bedside-Pipeline eine ideale Plattform." so Herold. Trotz mehrerer Alternativangebote entschied sich die Forscherin im Sommer, dem DZL treu zu bleiben. 
Rocio Sotillo - „Tackling chromosomal instability in lung cancer" Die Molekularbiologin Rocio Sotillo leitet seit Juli 2015 die neu gegründete Kooperationseinheit „Molekulare Grundlagen thorakaler Tumoren“, eine Brückeneinheit zwischen dem Deutschen Krebsforschungszentrum (DKFZ) und der Thoraxklinik am Universitätsklinikum Heidelberg (beide DZL-Standort TLRC). Zuvor war Rocio Sotillo am European Molecular Biology Laboratory (EMBL) in Monterotondo (Italien) tätig. Sotillo und ihr Team interessieren sich im Bereich Lungenkrebs insbesondere für Proteine, die auf Aneuploidie und chromosomale Instabilität (CIN) Einfluss nehmen, wie z.B. Mad2. Dieses Protein ist im menschlichen Tumorgewebe oft hochreguliert. „Um besser zu verstehen, ob die durch hohe Konzentrationen von Mad2 verursachte chromosomale Instabilität eine Rolle in der Tumorgenese spielt, haben wir den Effekt der Überexpression von Mad2 in Kombination mit dem Onkogen K-ras untersucht, einem Gen mit besonders hoher Mutationsrate im Lungenkrebs. Wir konnten zeigen, dass aneuploide Lungentumore nach zunächst erfolgreicher Therapie häufiger wieder auftreten“, erklärt Sotillo. Mit dem 2012 erhaltenen ERC Starting Grant konnte Rocio Sotillo für ihre Forschung am EMBL in Monterotondo moderne genomische Techniken einsetzen, um Modellsysteme zu entwickeln, die chromosomale Instabilität mit den für die Tumorgenese wichtigen Genen kombinieren. Der Wechsel an das DKFZ und das TLRC in Heidelberg ist eine wichtige und seit Langem angestrebte Gelegenheit für Sotillo, die über die letzten Jahre herangereiften Grundlagenforschungsprojekte in translationalen Projekten weiterzuentwickeln. „Unser Ziel ist es, das Verständnis zur Rolle von CIN in der Tumorprogression und dem Rezidiv zu verbessern und Mechanismen der Therapieresistenz zu identifizieren. Eventuell noch wichtiger scheint mir die Herausforderung für den Lungenkrebs zielgerichtete Therapien zu entwickeln, die Therapieresistenz schon im Ansatz berücksichtigen. Es ist essen-

\section{DZL-Publikationen}

Wöchentlich aktuelle Publikationen von DZL-Wissenschaftlern finden Sie unter der Rubrik „New in PubMed“: www.dzl.de/index.php/en/research/ publications/new-this-week-in-pubmed

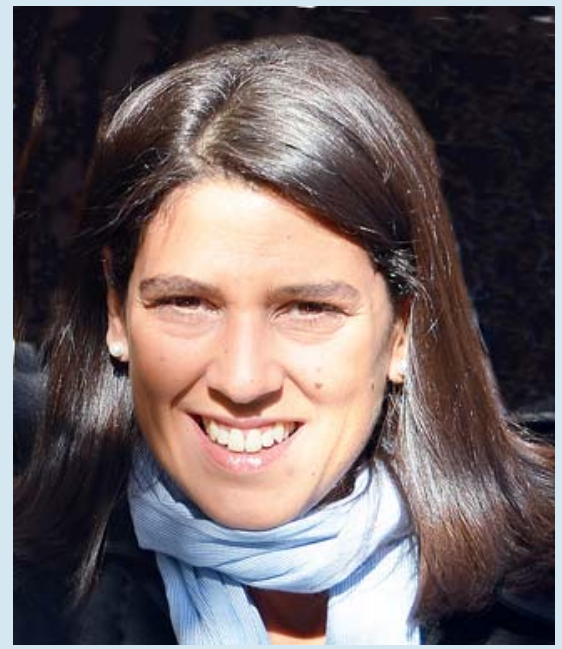

Medicine“ publiziert und auf der diesjährigen internationalen Jahrestagung der „American Thoracic Society“ (ATS) in Denver (USA) präsentiert.

Publikation: Krug N, Hohlfeld JM, Kirsten AM, Kornmann O, Beeh KM, Kappeler D, Korn S, Ignatenko S, Timmer W, Rogon C, Zeitvogel J, Zhang N, Bille J, Homburg U, Turowska A, Bachert C, Werfel T, Buhl R, Renz J, Garn H, Renz $\mathrm{H}$. Allergen-induced asthmatic responses modified by a GATA3-specific DNAzyme. N Engl J Med. 2015 May 21; 372 (21):1987-95. doi: 10.1056/NEJMoa1411776. Epub 2015 May 17.

$s b$

Abb.5 DZL-Nachwuchswissenschaftlerin Prof. Dr. Rocio Sotillo (Foto: R. S. privat).

tiell $\mathrm{zu}$ verstehen, auf welche Art und Weise CIN die Wirkung der Therapien beeinflusst, um effektivere Weg zur Bekämpfung des Lungenkrebs zu finden“. Die Kooperationseinheit zeichnet sich auch dadurch aus, dass Grundlagenwissenschaftler von der engen Zusammenarbeit mit den Klinikern der Thoraxklinik und der klinischen Kooperation innerhalb des DZL profitieren und, dass die translationale Forschung im Mittelpunkt steht.

\section{$b t$}

\section{Neuer Wirkstoff gegen allergisches Asthma $\nabla$}

Ein Team um die DZL-Wissenschaftler Prof. Dr. Harald Renz (Philipps-Universität Marburg/DZL-Standort UGMLC) und Prof. Dr. Norbert Krug (Fraunhofer ITEM/DZLStandort BREATH) hat einen neuartigen Wirkstoff gegen allergisches Asthma entwickelt und erfolgreich getestet: „SB010“, ein sogenanntes DNAzym hemmt mithilfe künstlicher DNA-Moleküle ein Protein, das für die typischen Asthmasymptome verantwortlich ist. Das DNAzym wirkt besonders zielgerichtet, wird inhalativ verabreicht und unterdrückt die Bildung des Transkriptionsfaktors GATA-3. In der erfolgreich abgeschlossenen Studie erwies sich das Präparat als sicher und gut verträglich. Es bereitet somit den Weg zu einem neuartigen Therapieprinzip insbesondere schwerer Verlaufsformen des allergischen Asthmas. Die Beschwerden der Asthmapatienten konnten um bis zu 34 Prozent abgeschwächt werden. Die Ergebnisse der klinischen Studie wurden im Fachmagazin „New England Journal of
Bei weiterem Interesse und Rückfragen zum DZL wenden Sie sich gerne an die DZL-Geschäftsstelle:

\section{Kontakt:}

DZL e.V.-Geschäftsstelle

Dr. Sylvia Weißmann

(kommissar. Geschäftsführerin)

Sabine Baumgarten, M. A.

(Projektkoordinatorin/Public Relations)

Aulweg 130, 35392 Gießen

Tel.: + 49 (0)641/9946721

E-Mail: s.baumgarten@dzl.de

www.dzl.de

\section{Termine}

2. - 3. Februar 2016

5. DZL-Jahrestreffen in Hannover

2 2. -5. März 2016

DZL beim 57. DGP-Kongress in Leipzig

-9. -12. April 2016

DZL und weitere Deutsche Zentren

beim Kongress der Deutschen

Gesellschaft für Innere Medizin (DGIM) in Mannheim

- 16. - 17. Juni 2016

5. Internationales DZL-Symposium in Hamburg

\section{Weitere Informationen im Internet}

- DZL-Homepage: www.dzl.de

- DZL-Kurzfilm: www.youtube.com/ watch?v=Ncbt_RSyu90

- Artikel zur Entstehung des DZL in The Lancet: www.thelancet.com/journals/lancet/article/PIIS0140-6736\%2812\% 2962013-3/fulltext

- Lungeninformationsdienst: www.lungeninformationsdienst.de 